\title{
Hypoglossal Facial Nerve Anastomosis for Post-Operative and Post-Traumatic Complete Facial Nerve Paralysis
}

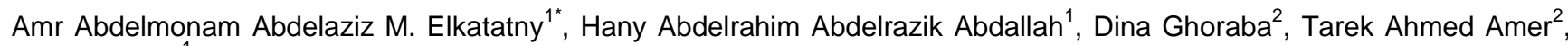 \\ Tarek Hamdy ${ }^{1}$ \\ ${ }^{1}$ Department of Neurosurgery, Kasr Alainy Medical School, Cairo University, Cairo, Egypt; ${ }^{2}$ Department of Plastic Surgery, \\ Kasr Alainy Medical school, Cairo University, Cairo, Egypt
}

\begin{abstract}
AIM: This study aims to evaluate the outcome of patients with complete facial paralysis following surgery to cerebellopontine angle tumours or following traumatic petrous bone fractures after reanimation by hypoglossalfacial anastomosis as regards clinical improvement of facial asymmetry and facial muscle contractility as well as complications associated with hypoglossal-facial reanimation procedure.

METHODS: This thesis included a prospective study to be carried out on 15 patients with unilateral complete lower motor neuron facial paralysis (11 patients after cerebellopontine angle tumour resection and 4 patients afte traumatic transverse petrous bone fracture) operated upon by end to end hypoglossal-facial nerve anastomosis in Cairo university hospitals in the period between June 2015 and January 2017.

RESULTS: At one year follow up the improvement of facial nerve functions were as follows: Three cases $(20 \%)$ had improved to House Hrackmann grade II, eleven cases $(73.33 \%)$ had improved to grade III, and one patient (6.66\%) had improved to House Brackmann grade IV.

CONCLUSION: Despite the various techniques in facial reanimation following facial nerve paralysis, the end to end hypoglossal-facial nerve anastomosis remains the gold standard procedure with satisfying results in cases of the viable distal facial stump and non-atrophic muscles. Early hypoglossal-facial anastomotic repair after acute facial nerve injury is associated with better long-term facial function outcomes and should be considered in the management algorithm.
\end{abstract}

Citation: Elkatatny AAAM, Abdallah HAA, Ghoraba D, Amer TA, Hamdy T. Hypoglossal Facial Nerve Anastomosis for Post-Operative and Post-Traumatic Complete Facial Nerve Paralysis. Open Access Maced Med Sci. 2019 (23):3984-3996 Keywords: Facial hypoglossal; Anastomosis; Facial palsy *Correspondence: Amr Abdelmonam Abdelaziz Mostaf Elkatatny. Department of Neurosurgery, Kasr Alainy Medical school, Cairo University, Cairo, Egypt. E-mal:

Received: 21-Apr-2019; Revised: 27-May-2019; Accepted: 28-May-2019; Online first: 29-Jul-2019

Copyright: (c) 2019 Amr Abdelmonam Abdelaziz M. Elkatatny, Hany Abdelrahim Abdelrazik Abdallah, Din Ghoraba, Tarek Ahmed Amer, Tarek Hamdy. This is an open-access article distributed under the terms of the Creative Commons Attibution-NonCommercial 4.0

Funding: This research did not receive any financia support

\section{Introduction}

The repair of skull bone defects is known as cranioplasty. Historically, such procedures date as early as BC 3000, where the Incas used gold to cover cranial defects resulting from trauma. There are several techniques for the repair of the cranial vault defects that can be broadly divided according to the graft used into autologous bone cranioplasty and allograft cranioplasty.

\section{Patients and Methods}

The present study was conducted prospectively on 15 patients with unilateral complete lower motor neuron facial paralysis (11 patients after cerebellopontine angle tumour resection and 4 patients after traumatic transverse petrous bone fracture) operated upon by end to end hypoglossalfacial nerve anastomosis in Cairo university hospitals in the period between June 2015 and January 2017.

\section{Inclusion criteria}

1. Patients with postoperative (following surgery to CPA tumours) and post-traumatic unilateral complete lower motor neuron facial nerve paralysis (House Brackmann grade VI) with intact both extracranial facial and hypoglossal nerves.

2. Time since injury is not more than 2 years, with intact facial mimetic muscles by E.M.G.

\section{Exclusion criteria}

1. Other causes of facial nerve paralysis. 
2. Partial facial nerve injuries, including House Brackmann grade $\mathrm{V}$ or less.

3. Patients with lower cranial nerve injuries. than 70 years.

4. Patients of age group less than 12 or more

5. Time since injury for more than 2 years.

6. Wasted non-functioning facial muscles guided by EMG.

7. Patients are not fit for surgery.

Preoperatively, all patients were subjected to the following:

History taking: - Age, sex, handedness, occupation; - History of present complaint; - Past medical history; - Drug history; - Family history and Social history.

Focusing on the history of previous trauma and clear fluid discharge from the affected ear or previous intracranial surgery, especially the skull base or CPA tumour resection.

\section{Examination}

General examination: this include assessment of vital signs (pulse rate, temperature, blood pressure and respiratory rate) and full examination of other body systems.

\section{Neurological examination}

1. General intellectual functions:

- Level of consciousness.

- Attentiveness

- Orientation: to self, place, time.

- Speech \& language: including fluency, repetition, comprehension, reading, writing, naming.

- Memory: including registration and retention.

- Higher intellectual functions: including general knowledge, abstraction, judgment, insight, and reasoning.

- Mood and affect.

2. Cranial Nerves:

- CN 1 - assess the smell

- CN 2 - visual acuity, visual fields

- CN 2 \& 3 pupillary response to light

- CN 3, 4, \& 6 - extra-ocular movements

- CN 5- sensory \& motor face; corneal examination):
Observing for asymmetry: By close attention to blinking, the nasolabial folds, and the corners of the mouth.

General examination: Otoscopy, palpation for masses near the neck and face, and examination of the skin.

Assessment of motor function: using House Backmann grading system, asking the patient to:

- Raise both eyebrows

- Close both eyes tightly

- Smile

- Puff out the cheeks

- Purse the lips

- Show both upper and lower teeth (grimace).

Assessment of special sensory function: Taste sensation of anterior two-thirds of the tongue.

Assessment of reflexes: Orbicularis reflex: tapping the glabella and observing asymmetry in blink pattern.

Bell's phenomenon: observe the upward movement of eyes during forced eye-closure

- CN8 - hearing

- CN 9 \& 10 - gag, palate rise

- CN 11 - neck turn/shoulder shrug

- CN12 - tongue movement. By looking for atrophy, weakness, or paralysis and fasciculations, and examination of associated dysarthria and dysphasia if present.

\section{Motor system examination:}

- muscle bulk

- muscle tone

- muscle power.

- reflexes, including superficial and deep reflexes.

\section{Sensory system examination:}

- Superficial sensation including fine touch, pain and temperature.

- Deep sensation including vibration sense, sense of position and movement and Romberg test.

\section{Coordination}

- finger to nose, heel to the shin, rapid alternating finger movements.

\section{Gait and sphincters.}

\section{Investigations}

A) Routine laboratory investigations including complete blood picture, bleeding profile, liver and renal functions, serum blood sugar as a part of pre- 
operative preparation.

B) Electromyography and Evoked Electromyography for assessment of degeneration, atrophy or regeneration of the facial nerve using needle electrodes placed in the target muscles that display action potentials during muscle contraction.

The face was examined at rest and during voluntary contraction.

C) Radiological investigations.

- Computerised tomography scan (CT): for assessment of petrous bone fracture in cases of posttraumatic facial nerve paralysis.

- Magnetic resonance imaging (MRI): for patients previously operated for cerebellopontine angle tumours to assess the degree of tumour resection and state of the facial nerve.

Table 7: House Brackmann scale for grading facial nerve paralysis

\begin{tabular}{|c|c|c|}
\hline \multirow[t]{2}{*}{ Grade } & \multirow[b]{2}{*}{ Normal } & \multirow{5}{*}{$\begin{array}{l}\text { Defined by } \\
\text { Normal facial function in all areas. } \\
\text { Slight weakness noticeable only on close inspection. At rest: normal symmetry } \\
\text { of forehead, ability to close eye with minimal effort and slight asymmetry, } \\
\text { ability to move corners of the mouth with maximal effort and slight asymmetry. } \\
\text { No synkinesis, contracture, or hemifacial spasm. } \\
\text { Obvious, but not disfiguring difference between two sides, no functional } \\
\text { impairment; noticeable, but not severe synkinesis, contracture, and/or } \\
\text { hemifacial spasm. At rest: normal symmmetry and tone. Motion: slight to no } \\
\text { movement of the forehead, ability to close eye with maximal effort and obvious } \\
\text { asymmetry, ability to move corners of the mouth with maximal effort and } \\
\text { obvious asymmertry. Patients who have obvious, but no disfiguring synkinesis, } \\
\text { contracture, and//or hemifacial spasm are grade iii regardless of the degree of } \\
\text { motor activity. }\end{array}$} \\
\hline & & \\
\hline$\|$ & Mild dysfunction & \\
\hline & & \\
\hline III & $\begin{array}{l}\text { Moderate } \\
\text { dysfunction }\end{array}$ & \\
\hline & & \\
\hline IV & $\begin{array}{l}\text { Moderate to } \\
\text { dysfunction }\end{array}$ & $v^{\text {and }} /$ or \\
\hline & & \\
\hline $\mathrm{v}$ & Severe $d$ & the \\
\hline & & \\
\hline VI & & \\
\hline
\end{tabular}

\section{Surgical technique}

the hypoglossal-facial anastomosis was performed under general anaesthesia in the supine position with the head tilted to the contralateral side.

Through an extended Lahey incision, the elevation of the anterior subplatysmal flap was done, the sternocleidomastoid muscle was retracted posteriorly and separated from the parotid gland; the facial nerve was identified at its exit from the stylomastoid foramen using the posterior belly of the digastric muscle and the tragal pointer as landmarks. The nerve was dissected of the parotid gland until the level of the pes anserinus.

Then the hypoglossal nerve was identified deep to the posterior belly of the digastric muscle and lateral to the internal carotid artery just above the carotid bifurcation, it was identified by following up the ansa hypoglossi up until it emerges from the hypoglossal nerve.

The nerve was cut as far distally as possible and mobilised after the transaction of the ansa hypoglossi. The facial nerve was transected at its exit from the stylomastoid foramen and reflected inferiorly; the ends of the two nerves were approximated without tension after cleaning of the epineurium and surrounding tissue to a limit of $5 \mathrm{~mm}$ from the ending cut.

Over a large gel foam piece, the ends of the nerves were anastomosed a traumatically using $3-4$ per neural sutures of 8-0 monofilament Nylon; a piece of fascia was wrapped around the anastomosis and secured with fibrin glue.

Preservation of digastric muscle was done because it gives protection to the anastomosis. After haemostasis, the closure was performed into two layers over a drain which was removed on the first postoperative day.

\section{Postoperative follow-up}

Post-operatively all the patients were admitted to an inward hospital room for 2 days with a suction drain that was removed day one after surgery, all patients received postoperative antibiotics and analgesics, and were discharged from the hospital 2-3 days following surgery.

\section{Postoperative} rehabilitation was done as follows:

- Mirror education (the patient compares the movement of the affected side to that of the normal side)

- Mirror exercises (the patient practices the desired movement, attempted bilaterally by watching the uninvolved side). This helps the patient to isolate and control the movement correctly before attempting it on the paretic side.

- Patients were learned to push the tongue against the teeth to produce a smile on the previously paralysed side.

Patients were followed up every 3 months with EMG, NCS and serial photography for one year. Patients were assessed regarding the following:

1. Improvement of facial symmetry and facial muscle contraction assessment using House Brackmann grading system.

2. Time elapsed for the appearance of facial movement (initial and complete).

3. Post-operative complications, including:

- Tongue paralysis complications including dysphagia (swallowing difficulty), dysarthria (speech difficulty), and hemiatrophy of the ipsilateral side of the tongue.

Synkinesis which mean involuntary movement occurring coordinated with a voluntary movement, and graded by 
Sunnybrook as follows:

1. Serial electrophysiological studies (EMG, NCS) at 3, 6, 9, 12 months, respectively.

All the data taken from the postoperative assessments were calculated, tabulated and statistically analysed.

\section{Post-operative rehabilitation}

The assistance of the patients in their goal of improved facial functions, treating them with facial neuromuscular retraining techniques, using a handheld mirror or surface EMG biofeedback. Treatment planning based on the evaluation findings and treatment-based categories. Treatment sessions one on one with a physical therapist for approximately 1 hour. A typical physical therapy session consisted of a brief re-evaluation, training with sEMG or a mirror, and instruction in an exercise program to be completed at home. Surface EMG biofeedback used initially to measure muscle activity associated with voluntary facial movements. Surface EMG biofeedback devices can be used to record and display small changes in muscle activity that cannot be seen in a mirror [1].

When the patient developed abnormal movement patterns or synkinesis, the surface EMG biofeedback again played an important role in the physical therapy session. Surface EMG biofeedback during exercise sessions helped to make patients more aware of the abnormal movement. Placement of the recording electrodes over the levator labii muscle (snarl muscle) and the inferior oculi muscle (eye closure). The patient would practice snarling and raising the line on the sEMG biofeedback screen associated with the levator labii muscle activity while keeping the activity from the inferior oculi muscle to a minimum. The sEMG biofeedback would often record activity in the inferior oculi muscle before any visible eye closure, providing the patient with the information necessary to correct his movements [2].

\section{Initiation}

Based on the initial signs and symptoms (severe resting asymmetry, minimal voluntary movement, absent synkinesis, and impaired function). Exercises typical for the initiation include an activeassisted range of motion exercises, during which the patient used the fingers of her hand to position a part of her face at a position in the range of motion for a specific movement and tried to hold the position using the targeted facial muscle while removing the passive assist. Often, patients find that it is easier to hold a position with a muscle than it is to move to the desired position (e.g. having a patient passively raise the involved eyebrow with a hand, then slowly removing the passive assist as the patient tries to activate the frontalis muscle and maintain the brow raise).
Because the patient can not voluntarily close his eye and has signs and symptoms of corneal irritation typical of patients in the initiation category, exercises focusing on closing the eye. Squinting or raising the lower eyelid was also included in the home facial exercise program. The patient is instructed to focus both eyes on an object positioned $30.5 \mathrm{~cm}(12$ in) down and in front of the patient and then to attempt to close both eyes. The eyes are to remain focused on this point until they are closed. Focusing the eyes downward helps to initiate the lowering of the upper eyelid. Maintaining the focused position until the eyes are closed prevents the Bell reflex, which can trick the patient into thinking that the eye is closed [3].

Muscle fatigue is often a concern when a patient is learning to initiate facial movements. To help avoid fatigue, the patient is instructed to do 5 to 10 repetitions of the facial exercises (smile, pucker, brow raise, frown, and eye closure) 3 times a day. The number of exercises kept to a minimum ( 3 - 5 exercises), as stated by Brach et al. .1999 [1].

\section{Facilitation}

Voluntary movement measured by the House Brackman facial grading system increases to minimal than to moderate movement. The patient initiates movement with mid-excursion and moderate asymmetry for all facial movements, and there is no evidence of synkinesis (sunny brook synkinesis score $=0)$.

Based on the increased voluntary movement and absent synkinesis, the patient considered to be in the facilitation category of treatment. The patient instructed inactively and resisted facial movement exercises typical for patients with some movement, no abnormal movement, and no difficulty with activities of daily living. Patient instructed to do symmetrical active facial movements without allowing the voluntary movement of the uninvolved side of the face to distort the movement of the involved side of the face. Maintaining symmetry is an important part of facial movement exercises. When the uninvolved facial muscles overpower the involved facial muscles, the facial posture tends to shift to the uninvolved side. When the facial posture shifts, the involved muscles are placed at a less-than-optimal length for the functioning (stretched). By maintaining symmetry and a more optimal length of the involved facial muscles during voluntary facial movements, we believe that the involved muscles have a better chance of functioning [1].

When some active movements are difficult to perform, such as lowering the bottom lip, functional activities, such as saying specific sounds, are used for exercise. The activity of lowering the bottom lip is an important component of saying words that begin with the letter " $F$ " patient-reported practising a word list to be easier than doing lip movement exercises, 
presumably because of his greater familiarity with the word task than with isolated oral movements.

Resistive facial exercises may be appropriate if the patient has no signs of synkinesis. Manual resistance is applied in the opposite direction of the desired movement. Resistance should be applied to only isolated facial movements, without causing mass action or synkinesis. Care must be taken not to over strengthen the uninvolved facial muscles, which would cause an even greater imbalance. An example of resistive facial exercises would be for the patient to provide resistance to the upper lip with a finger while attempting to pucker.

Facial muscle fatigue is no longer a primary concern when the patient is in the facilitation category. The patient is instructed to do a large number of repetitions $(10-20)$ of active or resistive exercises 1 to 2 times a day. Again, the number of exercises is limited to 3 to 5 to keep the patient-focused on the area needing the most work and to improve adherence to the exercise program. A typical exercise program for patients at this time would be 10 to 20 repetitions of 3 to 5 exercises to be completed 1 to 2 times a day [1].

\section{Movement Control}

Seven months after the initiation of therapy and 11 physical therapy sessions, most patient's resting posture changed from a drooping brow, lower eyelid, cheek, and mouth corner to a raised lower eyelid and a retracted cheek and mouth corner.

The patient's facial functioning continued to improve. Most patients have no problems with eating or performing oral hygiene (brushing her teeth).

Based on the appearance of inappropriate muscle activity and the presence of abnormal movement, the patient is now considered to be in the movement control category of treatment, with the facilitation category a secondary classification. Exercises focused on controlling the abnormal or synkinetic movement, such as raising the brow while keeping the eye open and controlling the ocular synkinesis. Movement control facial exercises emphasise moving only as much as the patient can without triggering the abnormal facial movement. The range of the movement is increased as long as the abnormal movement is controlled. The patient is told to concentrate on the quality of the exercise and not the quantity of the exercises completed. It is better for a patient to do 5 repetitions of an exercise correctly than it is to do 20 repetitions incorrectly [1].

Because facial muscle tightness often accompanies synkinesis, it is important to teach the patient facial muscle stretching exercises. The patient instructed in a stretching exercise that consisted of placing his right thumb inside his mouth, grasping the left cheek, and pulling the cheek down and across his face, thus applying a stretch to the cheek musculature. The stretch held for 20 seconds. The patient instructed to stretch her cheek 2 to 3 times, twice a day, to prevent shortening of muscle tissue. He instructed to stretch his cheek more often if he experienced cheek muscle tightness throughout the day.

Strengthening exercises for specific movements continued as long as they did not cause synkinesis. Patients instructed to continue with 2 to 3 of the strengthening exercises (10 -20 repetitions), 1 to 2 times a day, as explained in the "Facilitation" section [1].

\section{Results}

The study was conducted prospectively on 15 patients with unilateral complete lower motor neuron facial nerve paralysis operated upon by end to end hypoglossal-facial nerve anastomosis at Kasr El-Aini hospitals in the period from June 2015 to January 2017.

\section{Age and sex}

This study included 15 cases $9(60 \%)$ males and $6(40 \%)$ females, with male to female ratio of $3: 2$ (Figure 1).

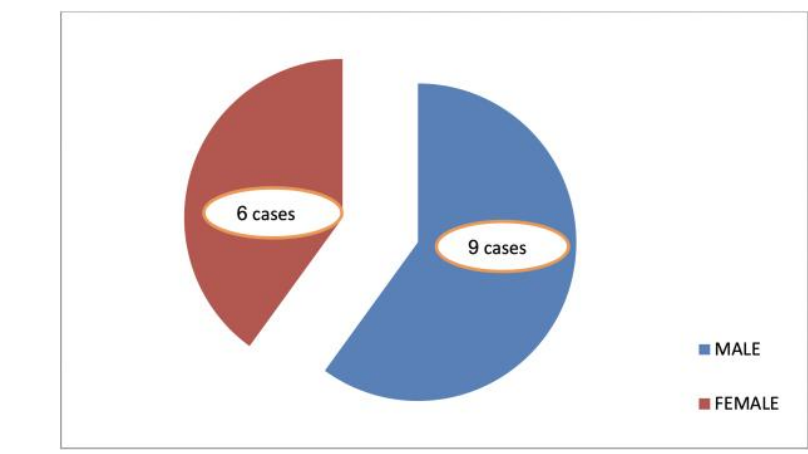

Figure 1: Histogram showing sex distribution

Their ages ranged from 22 to 58 years (4 patients between 20-30 years, 5 between 30-40 years, 1 between 40-50 years and 5 between 50-60 years) with a mean of $37.67 \pm 10.7$ years (Table 1 ).

Table 1: Percentage of patients in different age groups

\begin{tabular}{lcc}
\hline Age & Number of patients & \% Percentage \\
\hline $20-30$ & 4 & $26,66 \%$ \\
$30-40$ & 5 & $33,33 \%$ \\
$40-50$ & 1 & $6.66 \%$ \\
$50-60$ & 5 & $33.33 \%$ \\
\hline
\end{tabular}

\section{Aetiology}

$11(73.33 \%)$ of the cases were iatrogenic after removal of cerebellopontine angle tumours, and 4 
$(26.66 \%)$ cases were post-traumatic after severe head trauma that resulted in a petrous bone fracture and concomitant complete facial paralysis (Figure 2, left).

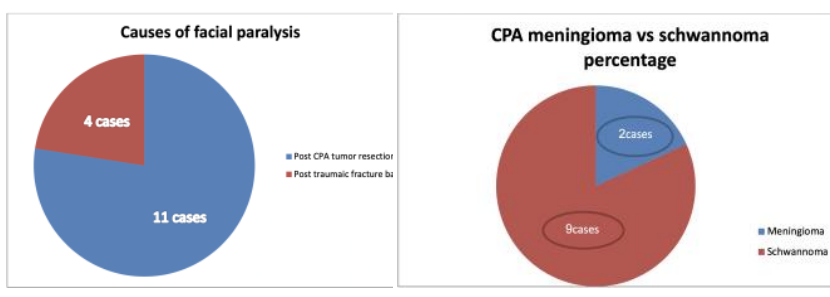

Figure 2: Histogram showing the aetiology of facial paralysis pre operatively (left); Showing the percentage of different CPA lesions preoperatively (right)

Two (13.33\%) of the eleven (73.33\%) CPA cases were meningioma, and nine were vestibular schwannoma (Figure 2, right).

\section{Side of the paralyzed facial nerve}

Paralysis includes the right facial nerve in 10 $(66.66 \%)$ cases, while the left facial nerve was involved in 5 (33.33\%) cases (Figure 5).

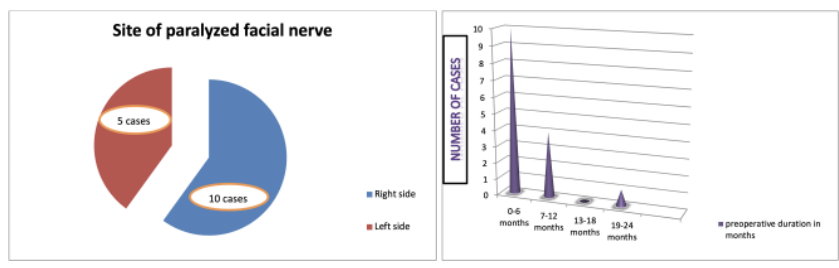

Figure 3: Histogram showing the percentage of affected side (left); Histogram showing the pre-operative duration of facial paralysis (right)

\section{Duration of facial nerve paralysis}

The duration of facial nerve paralysis before surgery ranged from 1 to 24 months, with a mean of 13 months.

The preoperative duration of facial nerve paralysis ranged from 1-6 months in 10 (66.66\%) patients, $7-12$ months in $4(26.66 \%)$ patients and 1924 months in 1 patient $(6.66 \%)$ (Figure 3, right).

\section{Clinical presentation}

Clinically all patients were complaining of complete lower motor neuron facial nerve paralysis classified according to the House Brackmann grading system as grade VI.

Thirteen $(86.66 \%)$ of the 15 cases were associated with $8^{\text {th }}$ cranial nerve injury (Figure 4 , left).

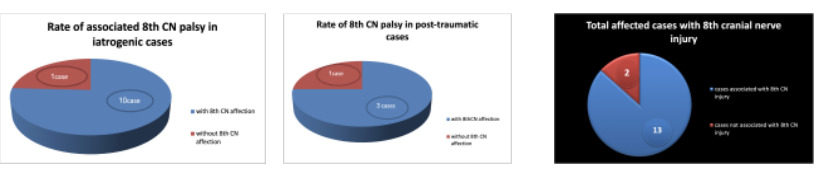

Figure 4: Histogram showing the rate of associated $8^{\text {th }} \mathrm{CN}$ in iatrogenic cases (left); Histogram showing the rate of 8th $\mathrm{CN}$ in post-traumatic facial paralysis (middle); Histogram showing a total number of affected cases with $8^{\text {th }} \mathrm{CN}$ injury (right)

Ten $(90.90 \%)$ of the eleven iatrogenic cases were associated with $8^{\text {th }}$ cranial nerve affection in the form of sensorineural hearing loss(Figure 4, middle).

And three (75\%) of the four post-traumatic patients were associated with $8^{\text {th }} \mathrm{CN}$ affection (Figure 4, left).

\section{Results}

At one year follow up, the improvement of facial nerve functions were as follows:

Three cases $(20 \%)$ had improved to House Hrackmann grade II, eleven cases (73.33\%) had improved to grade III, and one patient $(6.66 \%)$ had improved to House Brackmann grade IV (Figure 5).

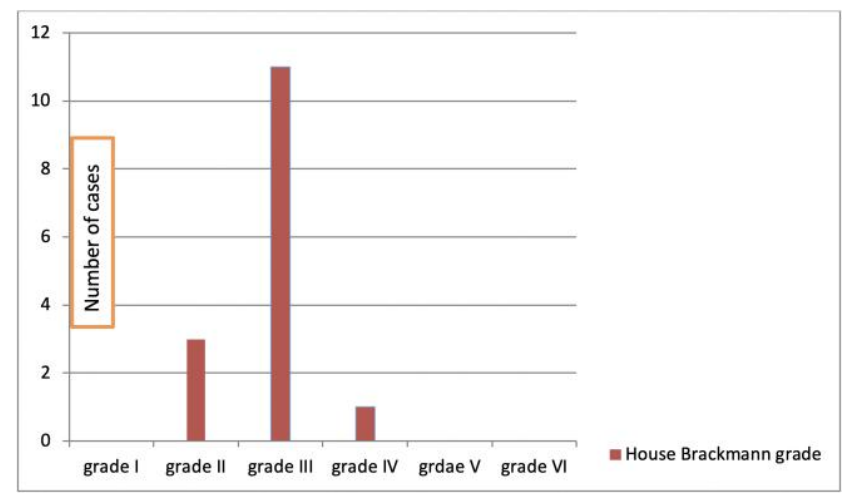

Figure 5: Histogram showing post-operative House Brackmann score and number of cases correlated

\section{Complications}

Hemiatrophy of the tongue occurred in 11 $(73.33 \%)$ cases, the atrophy was associated with difficulty in swallowing (dysphagia) in all 11 (100\%) cases affected and difficulty in speech (dysarthria) in 7 $(63.63 \%)$ of cases (Figure 6, left).

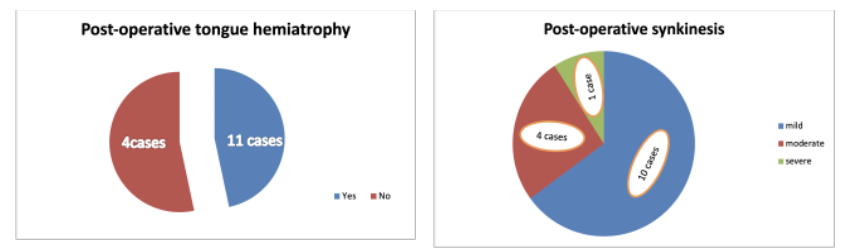

Figure 6: Histogram showing the number of cases complicated with tongue hemiatrophy (left); Histogram showing the percentage of post-operative synkinesis (right) 
degrees of synkinesis (the abnormal involuntary facial movement that occurs with voluntary movement of a different facial muscle group). Common types of facial synkinesis that occurred were oral-ocular synkinesis, involuntary eye closure during voluntary mouth movement, and ocular-oral synkinesis, involuntary mouth movement during voluntary eye closure, 10 $(66.66 \%$ ) cases were classified as mild ( according to Sunny brook grading system), $4(26.66 \%)$ cases as moderate, and $16.66 \%$ ) case as severe synkinesis (Figure 6, right).

All cases underwent neurophysiological monitoring at intervals of 3 months in between and results of average records were displayed as follows:

At 3 months: - The spontaneous pathological activity disappeared in all patients; - MUPs recorded in 7 patients.

At 6 months: - As previous record plus the MUPs recorded in 12 totals of 15 patients; Polyphasic regeneration potentials appeared.

At 9 months: - The previous record plus increased MUPs amplitude to the value of control muscles.

At 12 months: - The previous record and MUPs record in all 15 patients, and showing significantly increased duration above normal values; No reported mortality nor severe morbidity were recorded throughout the long term follow up period.

\section{Case 1}

\section{History}

50 years old female patient with a history of a motor car accident 8 months before admission that resulted in left transverse petrous bone fracture with clinical complete left lower motor facial nerve paralysis and CSF leakage (that stopped spontaneously after 3 days).

\section{Examination}

- Complete lower motor left facial nerve paralysis House Brackmann grade VI.

- Loss of hearing at the left ear.

\section{Investigations}

Radiological investigations were done, including CT scan, MRI of CPA and petrous bone which revealed a transverse petrous bone fracture in axial CT scan.

$\begin{array}{ccr}\text { Electromyography } & \text { and } & \text { Evoked } \\ \text { Electromyography revealed } & \text { action fibrillation }\end{array}$

potentials during muscle contraction.

Pure tone audiometry was done and revealed complete sensorineural hearing loss with no word discrimination.

\section{Surgery}

End to end left hypoglossal-facial nerve anastomosis 8 months after injury.

\section{Follow up}

One year following surgery, the facial nerve functions improved to House Brackmann grade III.

She had post-operative mild synkinesis and temporary tongue affection (mild dysphagia, dysarthria, and hemiatrophy) that improved 6 months later.

Post-operative EMG, NCS was done at three months intervals and revealed the following:

At 3 months: - The spontaneous pathological activity disappeared; - MUPs recorded.

At 6 months: - Polyphasic regeneration potentials appeared.

At 9 months: - The previous record plus increased MUPs amplitude to the value of control muscles.

At 12 months: - MUPs revealed significantly increased duration above normal values.

At one year follow up (Figure 7): Improvement of facial symmetry to grade III; Complete left sensorineural hearing loss that did not improve; - Mild hemiatrophy of the tongue, with improved dysarthria and dysphasia; - Mild synkinesis that improved at 12 months.

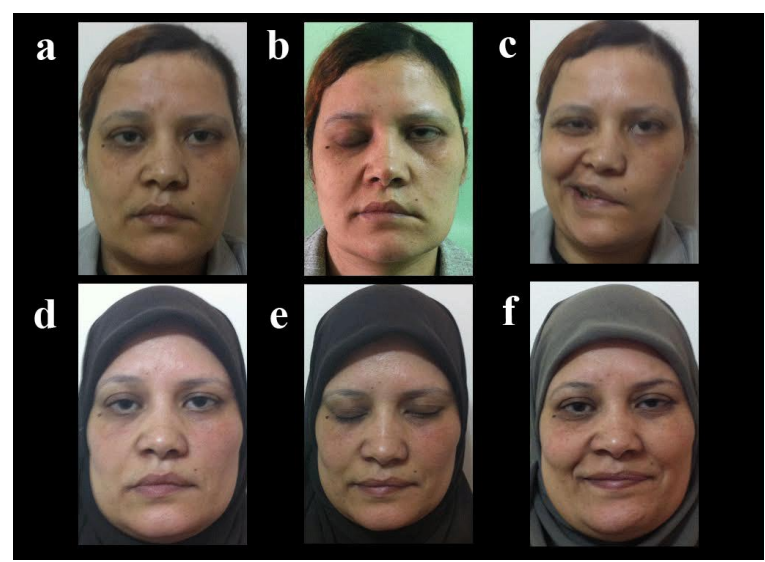

Figure 7: Patient with complete left facial paralysis: A) Preoperative resting view; $B$ ) Preoperative view on eye closure; $C$ ) Preoperative view on smiling; D) postoperative resting view; E) postoperative view on eye closure; F) Postoperative view on smiling 
Case 2

\section{History}

A 35 years old male patient with a history of right vestibular schwannoma resection 3 months before admission followed by right complete lower motor facial nerve paralysis and right severe sensorineural hearing loss.

\section{Examination}

- Complete lower motor right facial nerve paralysis House Brackmann grade VI.

- Diminution of hearing in the right ear. retrosigmoid incision)

- Scar of the previous operation (right

\section{Investigations}

Radiological investigations were done, including CT scan, MRI of brain and CPA, which revealed total tumour resection.

Electromyography and Evoked Electromyography revealed action fibrillation potentials during muscle contraction.

Pure tone audiometry was done and revealed complete sensorineural hearing loss with no word discrimination.

\section{Surgery}

End to end right hypoglossal-facial nerve anastomosis 3 months after injury.

\section{Follow up}

One year following surgery, the patient regained the facial nerve function of House Brackmann grade III.

He had post-operative moderate synkinesis, and temporary tongue affection (moderate dysphagia and dysarthria) that improved 7 months later and right tongue hemiatrophy that did not improve.

Post-operative EMG, NCS was done at three months intervals and revealed the following:

At 3 months: - The spontaneous pathological activity disappeared; - MUPs recorded.

At 6 months: - Polyphasic regeneration potentials appeared.

At 9 months: - The previous record plus increased MUPs amplitude to the value of control muscles.

At 12 months: - MUPs showing significantly increased duration above normal values.

At one year follow up (Figure 8): Improvement of facial symmetry to grade III; Complete right sensory neural hearing loss that did not improve; - Mild hemiatrophy of the tongue, with improved dysarthria and dysphasia; - Improvement of synkinesis from moderate to mild degree.

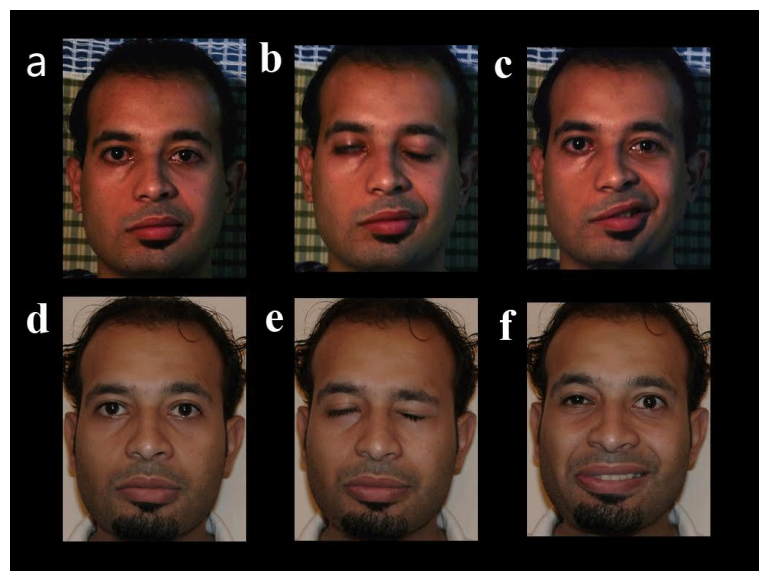

Figure 8: Patient with complete left facial paralysis; A) Preoperative resting view; $B)$ Preoperative view on eye closure; $C$ ) Preoperative view on smiling; D) Postoperative resting view E) Postoperative view on eye closure; F) Postoperative view on smiling

\section{Case 3}

\section{History}

A 39 years old male patient with a history of right CPA meningioma resection 7 months before admission followed by right complete lower motor facial nerve paralysis and diminution of hearing in the right ear.

\section{Examination}

- Complete lower motor right facial nerve paralysis House Brackmann grade VI.

- Diminution of hearing in the right ear.

- Scar of the previous operation (right retrosigmoid incision)

\section{Investigations}

Radiological investigations were done, including CT scan, MRI of brain and CPA, which revealed total tumour resection.

Electromyography and Evoked Electromyography revealed action fibrillation potentials during muscle contraction.

Pure tone revealed incomplete sensorineural hearing loss with moderate word discrimination. 


\section{Surgery}

End to end right hypoglossal-facial nerve anastomosis 7 months after injury.

\section{Follow up}

One year following surgery, the facial nerve functions improved to House Brackmann grade III. He had post-operative moderate synkinesis, and without tongue affection (no dysphasia nor dysarthria) and there were no tongue hemiatrophy observed.

Post-operative EMG, NCS was done at three months intervals and revealed the following:

At 3 months: - The spontaneous pathological activity disappeared; - MUPs recorded.

At 6 months: - Polyphasic regeneration potentials appeared.

At 9 months: - The previous record plus increased MUPs amplitude to the value of control muscles.

At 12 months: - MUPs revealed significantly increased duration above normal values.

At one year follow up (Figure 9): Improvement of facial symmetry to grade III; - Partial sensorineural hearing loss that was improving; - No hemiatrophy of the tongue, and no dysarthria nor dysphasia; - Synkinesis improved from moderate to a mild degree.

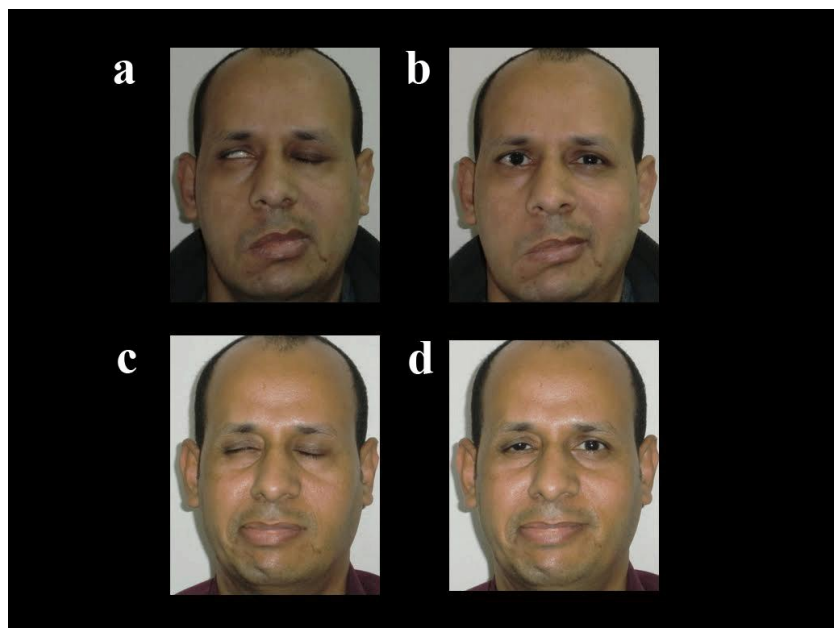

Figure 9: patient with complete left facial paralysis: a) preoperative view on eye closure b) preoperative view on smiling $c$ ) postoperative view on eye closure d) postoperative view on smiling

\section{Case 4}

\section{History}

Twenty-five years old male patient with a history of surgery for CPA meningioma resection that was extending to the middle cranial fossa, 1 month before admission followed by right complete lower motor facial nerve paralysis and right moderate sensorineural hearing loss.

\section{Examination}

- Complete lower motor right facial nerve paralysis House Brackmann grade VI.

- Diminution of hearing at the right ear.

- Scar of the previous cranial operation (combined right retrosigmoid and subtemporal incision)

\section{Investigations}

Radiological investigations were done, including CT scan, MRI of the brain and CPA, which revealed subtotal tumour resection.

\section{Electromyography and Evoked} Electromyography revealed action fibrillation potentials during muscle contraction.

Pure tone audiometry was done that revealed incomplete sensorineural hearing loss with moderate word discrimination.

\section{Surgery}

End to end hypoglossal-facial nerve anastomosis 1 month after injury.

\section{Follow up}

One year after surgery, he regained facial nerve functions to House Brackmann grade II.

He had post-operative mild synkinesis, severe tongue affection in the form of dysphagia, dysarthria and tongue hemiatrophy.

Post-operative EMG, NCS was done at three months intervals and revealed the following:

At 3 months: The spontaneous pathological activity disappeared; MUPs recorded.

At 6 months: Polyphasic regeneration potentials appeared; At 9 months; The previous record plus increased MUPs amplitude to the value of control muscles.

At 12 months: MUPs revealed significantly increased duration above normal values.

At one year follow up (Figure 10): Improvement of facial nerve function to grade II; Partial sensorineural hearing loss that was improving; Severe hemiatrophy of the tongue, dysarthria and dysphasia; Mild synkinesis that improved. 

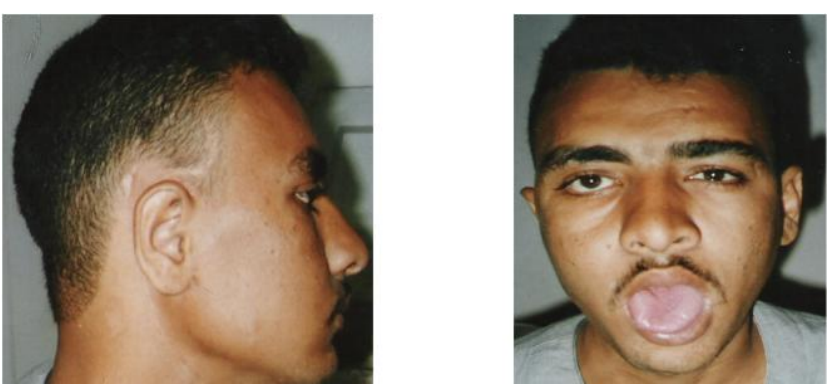

Figure 10: Postoperative view of a patient operated by right hypoglossal-facial nerve anastomosis, showing the scars of previous middle cranial fossa and hypoglossal-facial nerve anastomosis $(A)$ and tongue affection $(B)$.

\section{Discussion}

Peripheral facial nerve paralysis may be secondary or may not have a detectable cause (Bell's palsy). Three-quarters of Peripheral facial nerve paralysis is primary and one-quarter secondary. The most prevalent causes of secondary Peripheral facial nerve paralysis are systemic viral infections, trauma, surgery, diabetes, local infections, tumour, immunological disorders, or drugs [4].

Facial nerve paralysis is one of the most devastating nerve injuries with the important functional and psychological impact on the patient's life [5] Among the various aetiologies, Facial nerve indeed remains one of the major complications of cerebellopontine angle tumours surgery, and though its incidence has significantly diminished in recent decades due to advances in microsurgery and neuromonitoring and reported between $3 \%$ and $19 \%$ of major modern series [6], with the increase of traffic accidents, traumatic facial paralysis has also increased [7].

A multitude of reconstructive techniques using muscle transfers, free muscle grafts, shortening of weakened muscles, dermal transplants, fascial transplants, and the removal of redundant skin, have been described for reanimation of the paralysed face [8].

Several donor nerves are at the surgeon's disposal. Great auricular, sural, or medial and lateral antebrachial cutaneous nerves are all easily obtained [9], [10].

The hypoglossal-facial nerve graft has established itself over the years in an ever-increasing degree as an important method for restoration of the function of the mimetic facial muscles [11].

The end-to-end hypoglossal-facial anastomosis is the method of choice for facial nerve reconstruction. In cases with a long nerve defect, a cable graft interposition or facial nerve rerouting are indicated; for a tensionless anastomosis, the graft should be harvested $25 \%$ longer than the defect [12],[13].

The aim of our study was evaluating the outcome of patients with unilateral complete facial paralysis following surgery to cerebellopontine angle tumours or following traumatic petrous bone fractures after reanimation using hypoglossal-facial anastomosis end to end technique as regards clinical improvement of facial asymmetry and facial muscle contractility as well as complications associated with hypoglossal-facial reanimation procedure.

Our study was conducted prospectively on 15 patients with unilateral complete lower motor neuron facial nerve paralysis operated upon by end to end hypoglossal-facial nerve anastomosis at Kasr El-Aini hospitals in the period from June 2015 to January 2017. It included 9 (60\%) males and 6 (40\%) females, with male to female ratio of $3: 2$.

Male predominance was also found in the study of Flores in 2007 [14] which included 7 (87.5\%) males and $1(12.5 \%)$ females. On the other hand, female predominance was present in the study of Halil and his colleagues in 2006 [15] which included 9 $(69.2 \%)$ females and $4(30.8 \%)$ males and in the study of Samii in 2013 [16] which included 22 (55\%) females and 18 (45\%) males.

In our study, the age of the patients ranged from 22 to 58 years, with a mean of the mean age of $(37.67 \pm 10.7)$. The majority of the patients $(60 \%)$ were in the age range from $20-40$ years.

This is similar to the study of Hai-inn [7] where the mean age of the patients was 33years (range from 1 to 65 years). On the other hand, in the studies of Halil [15] and Pitty [13] the mean age of the patients was higher where the mean age was (44.5 years \{range 29-59\} and 47.3 years \{range 19 to 69\}) respectively.

In our study, 11 (73.33\%) of the cases were iatrogenic following resection of cerebellopontine angle tumours (9 $\{81.8 \%\}$ after vestibular schwannoma resection and $2 \quad\{18.2 \%\}$ after meningioma resection) while $4(26.66 \%)$ of the cases were post-traumatic following a petrous bone fracture.

This is Similar findings were reported 37 $(92.5 \%)$ of a total of 40 cases posted CPA tumour resection, especially vestibular schwannoma and 3 (7.5\%) were post-traumatic facial paralysis [16].

Conversely, Hai-jinn study stated that the most common cause was post-traumatic facial paralysis [7] where post-traumatic cases were 28 of $33(84.84 \%)$ patients while the iatrogenic cases were $5(15.15 \%)$ patients. However, it operated on 53-yearold woman presented with spontaneous complete (House Brackmann grade 6) facial palsy on her left side showed no improvement after 13 months of conservative treatment upon by hypoglossal-facial nerve anastomosis and improvement to House 
Brachman grade III occurred one year following surgery.

In our study the most common site affected was the right side, it was affected in $10(66.66 \%)$ of total 15 patients while the left side was affected in 5 (33.33\%) of patients.

Other studies did not focus on the side predominance in facial nerve paralysis [16], stated that there was no significant difference between right and left side on the rate of injury of facial nerve nor its improvement after reanimation. In our study, the duration of facial nerve paralysis before anastomosis ranged from 1-24 months where 10 (66.66\%) cases operated in the first 6 months of injury, 4 (26.66\%) between 6-18 months, and 1 (6.6\%) operated between 19-24 months. This is similar to the studies of Aboshanif [17] and (Hai-inn [7] where the preoperative duration of facial paralysis was less than 24 months (4 months and less than 2 years respectively). Other study had been operated on patients with facial paralysis for more than 2 years [18].

In our study, all patients had facial paralysis House Brackmann grade VI with loss of taste sensation of anterior two-thirds of affected side of the tongue ipsilateral to injured facial nerve, while the degree of pre-operative facial paralysis was of House Brackmann grade $\mathrm{VI}$ that reported in many previous studies [16], [17], [18]. On the other hand, Venail in 2009 [19] operated on 5 patients (45\% of his patients) with House Brackmann grade $\mathrm{V}$.

In our study, associated $8^{\text {th }}$ cranial nerve palsy was also present in $13(86.66 \%)$ of cases (10 of $11(90.90 \%)$ in iatrogenic cases and 3 of $4(75 \%)$ in post-traumatic petrous bone fracture cases). Also, Palombo [20] stated that there were a high percentage 20 of $33(60.60 \%)$ patients with some degree of hearing loss before and after the hypoglossal-facial anastomosis in a follow-up period of 15 years after surgery.

In this study, we assessed the facial muscles per-operatively with EMG, the presence of facial muscle fibrillation potentials favoured hypoglossalfacial nerve anastomosis while the presence of electrical silence indicated severe muscle atrophy which contraindicates the anastomosis.

In our study, all patients were operated upon by hypoglossal-facial nerve anastomosis; A cervical lazy S-shaped incision was made along the anterior edge of the sternocleidomastoid muscle. The sternocleidomastoid muscle was partially detached from the mastoid process and retracted posteriorly to expose the anterior one-third of the mastoid process, the facial nerve was exposed at the stylomastoid foramen and divided just distal to this point. The hypoglossal nerve was exposed medial to the tendon of the digastric muscle identified by the loop of the lingual artery and was divided. The proximal stump of the hypoglossal nerve was then anastomosed to the distal end of the facial (end to end technique), using fine a microstructure of 9-0 or 10-0 monofilament nylon. This end to end technique was adopted technique for management of complete facial paralysis by Venail in 2009 [19].

Other techniques were adopted for facial reanimation [21-23], like split (hemihypoglossal) with or without interposition jump graft technique. Also, another side to side technique where the hemihypoglossal-facial infratemporal side to side anastomosis were adopted and involves mobilisation of the intratemporal facial nerve, which is anastomosed to a partially incised hypoglossal nerve [24].

With an appropriate rehabilitation program, a satisfactory functional outcome can usually be achieved, but the sacrifice of the hypoglossal nerve unavoidably results in paralysis and atrophy of the ipsilateral tongue [18].

In our study patients regained satisfactory facial functions through 12 months duration as follows: 3 patients (20\%) improved to House Brackmann grade II, 11 patients (73.33\%) improved to grade III, and 1 patient $(6.66 \%)$ improved to House Brackmann grade IV. Most of our patients, who underwent postoperative rehabilitation, already had good facial symmetry at 12 months after surgery, with the reappearance of the nasolabial fold, Facial symmetry at rest as well as the closure of the eye. However, in the study by Linnet [25], only eight (34.7\%) out of 23 of their cases achieved grade III or better.

In our study, there were no significant difference in improvement of facial paralysis between different age groups where patients less than 40 years were 9 of total 15 cases ( 2 of them improved to House Brackmann grade II and 7 cases improved to grade III), and patients more than 40 years were 6 cases (1 case improved to grade II, 4 cases improved to grade and 1 case improved to grade IV), and this result was statistically insignificant.

Yetiser in 2007 [26], had meta-analyzed twenty-three articles about results of the hypoglossalfacial nerve anastomosis and he concluded that there was no significant difference in outcome between different age groups, and were also similar to reported earlier studies [16], [17], [18].

Conversely, Martins [22] stated that Better results are obtained in younger patients (15 of 35 $(41.66 \%)$ of his patients were less than 20 years) and with a shorter interval between facial nerve injury and surgery.

This study revealed no difference in outcome between patients with facial paralysis iatrogenic after CPA tumour resection and post-traumatic facial paralysis. The same results were obtained by Aboshanif [17] who conducted a study on 22 patients with either traumatic or iatrogenic complete facial 
nerve paralysis House Brackmann grade VI, 8 (36.36\%) of his patients regained facial nerve function as House Brackmann Grade III, and 3 cases (13.6\%) as grade IV, regardless the aetiology of facial paralysis.

In our study, the duration of facial nerve paralysis pre-operatively had a statistically significant effect on the results after surgical hypoglossal-facial anastomosis, all patients with a duration of 1-12 months facial nerve paralysis preoperatively achieved post-operative House Brackmann grade III or less while the only patient who was operated 19-24 months following injury achieved House Brackmann grade IV.

Good results were reported by Gagnon and his colleagues in 1989 [27] in five patients with paralysis of 2.5 to 7 years, and they reported that "gross intraoperative assessment for nerve atrophy was the best method for predicting successful reinnervation after anastomosis", but the number of cases in previously reported results of delayed reinnervation was very small to be satisfactory valid.

In our study, hemitongue atrophy that resulted from sectioning of the hypoglossal nerve was reported in $11(73.33 \%)$ of total 15 patients, manifested with difficulty in swallowing (dysphagia) in 7 (63.63\%) of 11 affected cases and speech (dysarthria) in all 11 $(100 \%)$ cases affected, and most of the patients improved within 12 months after surgery without affection of patient's quality of life after rehabilitation programs. The study of Halil [15] reported hemitongue atrophy in all $13(100 \%)$ patients of his study in different degrees (8 cases $\{53.33 \%\}$ of severe, 5 cases $\{38.46 \%\}$ moderate atrophy). Kunert [28] reported 3 of his 7 cases (42.85\%) operated upon by hemihypoglossal facial anastomosis (split technique) showed post-operative tongue hemiatriohy.

Also, we reported the facial synthesis of different degrees in all patients. Synkinesis (the abnormal involuntary facial movement that occurs with voluntary movement of a different facial muscle group). Common types of facial synkinesis are oralocular synkinesis, involuntary eye closure during voluntary mouth movement and ocular-oral synkinesis, involuntary mouth movement during voluntary eye closure, that occurred mainly during facial expression were $10(66.66 \%)$ cases had classified according to Sunny brook grading system as mild (when involuntary muscle contractions were slight during raising forehead, closure of the eye, opening of mouth, elevating nasal snare, and during lip bickering), $4(26.66 \%)$ cases of moderate (when there were slight synkinetic movement associated with disfigurement), and one 6.66\%) case of severe synkinesis (when there were disfiguring synkinesis and gross mass movement of several muscles). That also improved 12 months following surgery in all patients.
Fourteen patients out of thirty $(46.66 \%)$ developed mild synkinesis involving contraction of the orbicularis oculi muscle on activation of the orbicularis oris, zygomaticus major and risorius muscles; two such cases were treated with botulinum toxin injections in a previous study [29].

On the other hand, facial synkinesis was noted in 8 out of 60 patients $(13.33 \%$ ) in the previous study using the same technique [16], and one out of 12 patients (8.3\%) in another study [19] using a modified end to side technique whereas older studies preferred to anastomose a split hypoglossal nerve to the facial nerve [10], [30]. Also, a similar technique to perform an end-to-side anastomosis using a skeletonised intratemporal segment of the facial nerve, which is transected and mobilised towards the hypoglossal nerve was adopted previously [11],[18]

In our study, all cases underwent neurophysiological monitoring at intervals of 3 months in between, and the results of average records were displayed as follows:

At 3 months: - The spontaneous pathological activity disappeared in all patients; - MUPs recorded in 7 patients.

At 6 months: - As previous record plus the MUPs recorded in 12 total of 15 patients; - Polyphasic regeneration potentials appeared.

At 9 months: - The previous record plus increased MUPs amplitude to the value of control muscles.

At 12 months: - The previous record and MUPs record in all 15 patients, and showing significantly increased duration above normal values.

EMG plays a central role in the evaluation of the patients, as stated by Gerd in 2010 [31]. Muscular damage leads to alterations of the insertion potentials during needle EMG. EMG allows a prognosis on the probability of spontaneous healing [32]. In patients with regeneration and defective healing, the clinical examination, together with EMG allows the physician to evaluate the severity of dyskinesia, synkinesia, and autocatalytic syndrome [33]

Also, it was stated by Flasar in 2017 [34], that reinnervation can be detected at least 2 months earlier by EMG than by clinical evaluation. Changes should be followed for at least 18 months to assess the outcome. EMG changes reflected the remodelling of motor units due to axonal regeneration and collateral sprouting by hypoglossal nerve fibres into the reinnervated facial muscle fibres.

In our study, we used facial neuromuscular re-education techniques (i.e. the use of facial exercises to address a patient's impairments and functional limitations) as an exercise program for the rehabilitation of facial muscle functions that changed over time as the patient's impairments change with recovery. The facial neuromuscular re-education 
exercise program was emphasized accuracy of facial movement patterns and isolated muscle control, the number of exercise repetitions and the frequency of the exercise program depending on the treatmentbased categories, were based on the patient's impairments.

The same exercise technique was done with good results after 13 months of physiotherapy for a patient with bell's palsy [2].

\section{References}

1. Brach JS, VanSwearingen JM, Et al. Facial neuromuscular retraining for oral synkinesis. Plast Reconstr Surg. 1997; 99:1922-31. https://doi.org/10.1097/00006534-199706000-00017 PMid:9180715

2. Brach JS, VanSwearingen JM. Physical therapy for facial paralysis: a tailored treatment approach. Phys Ther. 1999; 79(4):397-404. https://doi.org/10.1093/pti/79.4.397

3. Jelks GW, Smith B, Bosniak S. The evaluation and management of the eye in facial palsy. Clin Plast Surg . 1979; 6:397-419.

4. Finsterer J. Management of peripheral facial nerve palsy. Eur Arch Otorhinolaryngol. 2008; 265(7):743-52. https://doi.org/10.1007/s00405008-0646-4 PMid:18368417 PMCid:PMC2440925

5. Samii M, Matthies $C$. Indication, technique and results of facial nerve reconstruction. Acta Neurochir. 1994; 130:125-39. https://doi.org/10.1007/BF01405512 PMid:7725935

6. Koerbel A, Gharabaghi A, Et al., Evolution of vestibular schwannoma surgery: the long journey to current success. Neurosurg Focus. 2005; 18(4):e10. https://doi.org/10.3171/foc.2005.18.4.11 PMid:15844862

7. YI Hai-jnn, LIU Pi-Nan, YANG Shi-ming. Surgical Management of Traumatic Facial Paralysis: A Case Review Study. Journal of Otology; 2011; (6) 2: 38-42. https://doi.org/10.1016/S1672-2930(11)50020-0

8. Rosenwasser $\mathrm{RH}$, Liebman $\mathrm{E}$, Et al. Facial reanimation after facial nerve injury. Neurosurgery. 1991; 29:568-74. https://doi.org/10.1227/00006123-199110000-00014 PMid:1944838 9. Humphrey CD, Kriet JD. Nerve repair and cable grafting for facial paralysis. Facial Plast Surg. 2008; 24(2):170-6. https://doi.org/10.1055/s-2008-1075832 PMid:18470828

10. Arai H, Sato K, Yanai A. Hemihypoglossal-facial nerve anastomosis in treating unilateral facial palsy after acoustic neurinoma resection. $J$ Neurosurg; 1995; 82: 51-54. https://doi.org/10.3171/jns.1995.82.1.0051 PMid:7815134

11. Atlas MD, Lowinger DS. A new technique for hypoglossal-facia nerve repair. Laryngoscope 1997; 107:984-91. https://doi.org/10.1097/00005537-199707000-00028 PMid:9217143 12. Falcioni $\mathrm{M}$, Taibah $\mathrm{A}$, Et al. Facial nerve grafting. Otol Neurotol. 2003; 24(3): 486-89. https://doi.org/10.1097/00129492-20030500000022 PMid: 12806304

13. Pitty LF, Tator $\mathrm{CH}$. Hypoglossal-facial nerve anastomosis for facial nerve palsy following surgery for cerebellopontine angle tumors. $J$ Neurosurg. 1992; 77:724-31.

https://doi.org/10.3171/ins.1992.77.5.0724 PMid:1403114

14. Flores LP. Surgical results of the Hypoglossal-Facial nerve Jump Graft technique. Acta Neurochir (Wien). 2007; 149: 1205-10. https://doi.org/10.1007/s00701-007-1412-x PMid:17978883

15. Halil AK, Galip Z, Mustafa O. Hypoglossal-Facial Nerve Anastomosis for Facial Nerve Palsy Following Surgery for Vestibular Schwannoma. Turkish Neurosurgery 2006; (16)4: 158-62.

16. Sami TH, David VK, Et al. Facial Reanimation After Facial Nerve Injury Using Hypoglossal to Facial Nerve Anastomosis: The Gruppo Otologico Experience. Indian J Otolaryngol Head Neck Surg. 2013;
65(4):305-8. https://doi.org/10.1007/s12070-011-0468-3 PMid:24427588 PMCid:PMC3851504

17. Aboshanif M, Eigo O, Et al. Outcome of different facial nerve reconstruction techniques. Braz J Otorhinolaryngol. 2016; 82(6):702-9. https://doi.org/10.1016/i.bjorl.2015.12.010 PMid:27222117

18. Sawamura Y, Abe H. Hypoglossal-facial nerve side-to-end anastomosis for preservation of hypoglossal function: results of delayed treatment with a new technique, J Neurosurg. 1997; 86(2):203-6. https://doi.org/10.3171/jns.1997.86.2.0203 PMid:9010420

19. Venail F, Sabatier P, Et al. Outcomes and complications of direct end-to-side facial-hypoglossal nerve anastomosis according to the modified May technique. J Neurosurg. 2009; 110(4):786-91. https://doi.org/10.3171/2008 9.JNS08769 PMid:19119877

20. Palombo AA, Shibukawa AF. Hearing loss in peripheral facial palsy after decompression surgery. Braz J Otorhinolaryngol. 2012; 78(3):216. https://doi.org/10.1590/S1808-86942012000300005 PMid:22714842 21. Kyung S, June-kyu K, Et al., Hypoglossal-facial crossover in facialnerve palsy: pure end-to-side anastomosis technique. British Journal of Plastic Surgery. 2002; (55)1: 25-31. https://doi.org/10.1054/bjps.2001.3727 PMid:11783965

22. Martins RS, Socolovsky M, Et al. Hemihypoglossal facial neurorrhaphy after mastoid dissection of the facial nerve: results in 24patients and comparison with the classic technique. Neurosurgery. 2008; 63(2):310-6.

https://doi.org/10.1227/01.NEU.0000312387.52508.2C PMid:1879736

23. Liu P, Liao C, Et al. Comparison of 4 Different Methods for Direct Hypoglossal-Facial Nerve Anastomosis in Rats. World Neurosurg. 2018; S1878-8750(18)30137-2.

24. Lassaletta L, González T. Hemi-hypoglossal-facial intratemporal side to side anastomosis. Acta Otorrinolaringol Esp. 2008; 59(3):124-6. https://doi.org/10.1016/S0001-6519(08)73279-5

25. Linnet J, Madsen FF. "Hypoglosso-facial nerve anastomosis," Acta Neurochirurgica. Acta Neurochir (Wien). 1995; 133(3-4):112-5. https://doi.org/10.1007/BF01420060 PMid:8748752

26. Yetiser S, Karapinar U. Hypoglossal-facial nerve anastomosis: a meta-analytic study. Ann Otol Rhinol Laryngol. 2007; 116(7):542-9. https://doi.org/10.1177/000348940711600710 PMid:17727086

27. Gagnon NB, Molina-Negro P. Facial reinnervation after facial paralysis: is it ever too late?. Arch Otorhinolaryngol. 1989; 246(5):303-

7. https://doi.org/10.1007/BF00463581 PMid:2590042

28. Kunert $P$, Podgórska A, Et al. Hemihypoglossal-facial nerve anastomosis for facial nerve palsy. Neurol Neurochir Pol. 2011; 45(5):452-60. https://doi.org/10.1016/S0028-3843(14)60313-3

29. Dalla TE, Furini F, Et al. Evaluation and treatment of synkinesis with botulinum toxin following facial nerve palsy. Disabil Rehabil. 2010; 32:1414-18. https://doi.org/10.3109/09638280903514697 PMid:20156046

30. Cusimano MD, Sekhar L: Partial hypoglossal to facial nerve anastomosis for reinnervation of the paralyzed face in patients with lower cranial nerve palsies: technical note. Neurosurgery. 1994; 35:532-34. https://doi.org/10.1227/00006123-199409000-00027 PMid:7800149

31. Gerd FV, Mira P, Orlando G. Modern concepts in facial nerve reconstruction. Head Face Med. 2010; 6: 25.

https://doi.org/10.1186/1746-160X-6-25 PMid:21040532

PMCid:PMC2984557

32. Grosheva M, Wittekindt C, Guntinas-Lichius O. Prognostic value of electroneurography and electromyography in facial palsy.

Laryngoscope. 2008; 118: 394-97.

https://doi.org/10.1097/MLG.0b013e31815d8e68 PMid:18090862

33. Guntinas-Lichius O, Streppel M, Stennert E. Postoperative functional evaluation of different reanimation techniques for facial nerve repair. AmJSurg. 2006; 191: 61-7.

https://doi.org/10.1016/i.amjsurg.2005.05.054 PMid:16399108

34. Flasar J, Volk GF, Et al. Quantitative facial electromyography monitoring after hypoglossal-facial jump nerve suture. Laryngoscope Investig Otolaryngol. 2017; 2(5): 325-30. https://doi.org/10.1002/lio2.95 PMid:29094077 PMCid:PMC5655551 